\title{
Estudo retrospectivo do comportamento da força muscular em pacientes com Síndrome Pós-Poliomielite
}

\author{
Retrospective study on the behavior of muscular strength of Post-Poliomyelitis Syndrome patients \\ Vanessa Lima', Fernanda Maggi², Abrahão Augusto Juviniano Quadros', \\ Acary Souza Bulle Oliveira ${ }^{4}$, Sissy Veloso Fontes ${ }^{5}$, Francis Meire Fávero ${ }^{6}$
}

\section{RESUMO}

Objetivos. Analisar o comportamento da força muscular (FM) dos membros superiores e inferiores mais funcionais de pacientes com Síndrome Pós Poliomielite (SPP), através da análise dos dados obtidos em duas avaliaçóes e verificar se houve variação da FM total nos membros estudados. Método. Estudo retrospectivo com coleta de dados de pacientes com SPP do Setor de Investigaçóes nas Doenças Neuromusculares (SIDNM) da UNIFESP/EPM. Foram coletados resultados de duas avaliaçóes do teste de FM manual classificado pela Medical Research Council (MRC) de 12 grupos musculares. Ao resultado final, foi aplicado o cálculo de porcentagem do teste MRC. Resultados. Não houve redução da FM avaliados pelo MRC entre a $1^{\text {a }}$ e $2^{a}$ avaliaçáo. Tendo como base a amostra total do estudo, observou-se diminuiçáo da FM de flexores de joelho. Quanto à FM total dos membros superiores, verificou-se que houve diminuição da FM total em três grupos de pacientes avaliados. Já nos membros inferiores, a FM manteve-se estável em três grupos avaliados. Conclusáo. Observaram-se pequenas variaçóes na FM dentro dos períodos acompanhados. A queixa da nova fraqueza muscular pode estar relacionada a outros fatores, como fadiga ou envelhecimento.

Unitermos. Síndrome Pós-poliomielite, Fraqueza Muscular, Força muscular, Reabilitação

Citaçáo. Lima V, Maggi F, Quadros AAJ, Oliveira ASB, Fontes SV, Fávero FM. Estudo retrospectivo do comportamento da força muscular em pacientes com Síndrome Pós-Poliomielite.

\begin{abstract}
Objective. To analyze the behavior of muscle strength (MS) of the upper and lower limbs more functional Post-Polio Syndrome (PPS) patients, through the analysis of data from two trials, check if there was variation of total MS limbs studied. Method. Retrospective data collection of patients with PPS at the Sector of Research in Neuromuscular Diseases (SIDNM) UNIFESP/EPM. We collected results of two test ratings of FM manual classified by Medical Research Council (MRC) of 12 muscle groups. At the end result, we applied the percentage calculation test MRC. Results. There was no reduction of MS assessed by MRC between 1st and 2nd assessment. Therefore, based on the total study sample, there was a decrease in MS knee flexors. As for MS upper limbs, it was found that there were decreases in MS total three groups of patients. Already in the lower limbs, the total MS remained stable in three groups. Conclusion. There was little variation in MS within the periods monitored. Hence, the complaint of new muscle weakness may be related to other factors, such as fatigue or aging.
\end{abstract}

Keywords. Postpoliomyelitis Syndrome, Muscle Weakness, Muscle Strength, Rehabilitation

Citation. Lima V, Maggi F, Quadros AAJ, Oliveira ASB, Fontes SV, Fávero FM. Retrospective study on the behavior of muscular strength of Post-Poliomyelitis Syndrome patients.

\footnotetext{
Trabalho realizado no Ambulatório de SPP do Setor de Investigaçáo de Doenças Neuromusculares da UNIFESP/EPM, Sáo Paulo-SP, Brasil.

1. Fisioterapeuta, pós-graduanda em Doenças Neuromusculares pela UNIFESP/EPM, São Paulo - SP, Brasil.

2. Fisioterapeuta, pós graduada em Doenças Neuromusculares pela UNIFESP/ EPM, São Paulo - SP, Brasil.

3. Fisioterapeuta, Doutor em Ciências da Saúde pela UNIFESP/EPM, São Paulo - SP, Brasil

4. Neurologista, Doutor, Professor Afiliado da Disciplina de Neurologia da UNIFESP/EPM, São Paulo - SP, Brasil.

5. Fisioterapeuta, Doutora em Ciências pela UNIFESP/EPM, São Paulo - SP, Brasil

6. Fisioterapeuta, Doutora, Coordenadora do Curso de Especializaçáo em Intervenção Fisioterapêutica nas Doenças Neuromusculares da UNIFESP-EPM, São Paulo - SP, Brasil.
}

Endereço para correspondência: Francis Meire Favero R. Estado de Israel, 899 - Vila Clementino CEP 04022-000, São Paulo-SP, Brasil ffave.nexp@latoneuro.com.br Aceito em: 11/08/14

Conflito de interesses: não 


\section{INTRODUÇÃO}

A Poliomielite anterior aguda é uma doença viral causada por um enterovírus que afeta predominantemente os neurônios motores inferiores localizados na medula espinal, constituindo uma paralisia flácida assimétrica de músculos da coluna vertebral e principalmente dos membros inferiores (MMII), e que representou, até 1960, a maior causa de paralisia motora e óbito em crianças e adultos jovens em todo o mundo ${ }^{1-3}$.

A Síndrome Pós-poliomielite (SPP) é caracterizada pelo aparecimento de novos sinais e sintomas neuromusculares em pessoas que foram acometidas pelo vírus da poliomielite aguda na infância, agravando as sequelas motoras da poliomielite. Estão presentes as novas manifestaçôes: fadiga anormal seja um cansaço muscular súbito ou uma sensação generalizada de exaustão; nova fraqueza muscular, podendo ser tanto dos músculos que foram originalmente afetados, quanto naqueles considerados clinicamente náo afetados; dores musculares e/ou articulares; perturbaçôes do sono; dificuldades respiratórias; disfagia; intolerância ao frio e redução da capacidade funcional, o que afeta de forma negativa a qualidade de vida desses indivíduos ${ }^{4-6}$.

Atualmente, existem estudos analisando a progressão da fraqueza muscular em pacientes com SPP, porém não foram encontrados estudos realizados neste sentido com a população brasileira ${ }^{7,8}$. Portanto, esta pesquisa consiste na investigação do comportamento da força muscular (FM) em pacientes com SPP e, desta forma, ajudar na informação dos profissionais da área da saúde sobre esta nova e frequente manifestação. Assim, para esta pesquisa foi analisado retrospectivamente o comportamento da FM dos membros superiores (MMSS) e MMII mais funcionais, ou seja, dos membros menos afetados dos pacientes com SPP, em diferentes intervalos de acompanhamento (dois, três, quatro e cinco anos), através da análise dos dados obtidos em dois testes de FM manual e verificar a FM total nos membros mais funcionais dos pacientes com SPP.

\section{MÉTODO}

\section{Local}

Os dados para o atual estudo retrospectivo foram coletados no Setor de Investigaçôes nas Doenças Neuro- musculares (SIDNM), Disciplina de Neurologia Clínica, Departamento de Neurologia/Neurocirurgia, Universidade Federal de São Paulo - UNIFESP/EPM. O estudo foi aprovado pela Comissão de Ética e Pesquisa da Universidade Federal de São Paulo - UNIFESP/Hospital São Paulo, número do parecer 203.093.

\section{Amostra}

Foram incluídos 12 pacientes com SPP, de ambos os sexos, com histórico confirmado de poliomielite infantil aguda, com diagnóstico confirmado de SPP, sendo inserido no protocolo SIDNM da UNIFESP, com faixa etária de 30 a 59 anos de idade, apresentando novo sintoma de fraqueza muscular a, pelo menos, um ano. Esses devem ter realizado acompanhamento do estado da FM por no mínimo dois anos e assinado o Termo de Consentimento Livre e Esclarecido usado no ambulatório da UNIFESP. Foram excluídos os pacientes de SPP que apresentaram doenças associadas como, doenças metabólicas descompensadas, neuronopatias e doenças neurológicas, aqueles que tinham história recente de cirurgias ortopédicas corretivas e indivíduos que relatam muitas dores ao realizar os movimentos. A caracterização da amostra foi realizada com a seleção de dados das fichas de avaliação fisioterapêutica da instituição de pacientes com SPP.

\section{Procedimento}

Os dados coletados da avaliação fisioterapêutica foram dados pessoais, dados sobre a história e recuperação da pólio aguda, novos sintomas, doenças associadas, exame físico e teste de FM manual classificados pela Medical Research Council MRC 9 que é aplicada com o objetivo de avaliação da FM na rotina do ambulatório de SPP, no qual são avaliados 35 grupos musculares nos seguimentos: cervical, tronco, MMSS e MMII, utilizando pontuaçôes inteiras e realizadas por um fisioterapeuta.

A amostra foi dividida em quatro grupos, de acordo com o tempo de intervalo entre o primeiro e o segundo teste de FM analisado. Cada grupo foi composto por três indivíduos. No grupo I, foram incluídos pacientes que tiveram dois anos de intervalo entre a primeira e a segunda avaliação, ou seja, foi realizado o primeiro teste de FM e, após dois anos, o mesmo paciente foi submetido a um novo teste de FM. No grupo II, fizeram parte os 
indivíduos com três anos de intervalo entre a primeira e a segunda avaliação; grupo III, com intervalo de quatro anos e o grupo IV, com intervalo de cinco anos.

Dos quatro grupos de pacientes incluídos no estudo foram recolhidos resultados retrospectivos de dois testes de FM classificado pela MRC, de 12 grupos musculares dos membros mais funcionais dos pacientes. Dos MMSS, foram avaliados três grupos musculares: abdutores do ombro, flexores de cotovelo e extensores de punho; dos MMII: flexores de quadril, extensores de joelho e dorsiflexores de tornozelos ${ }^{10-12}$.

Ao final, para a verificação da FM total do membro estudado, foi utilizado o seguinte cálculo:

FM total $(\% \text { MRC })^{13}=\underline{\text { Soma das médias do MRC } \times 100}$

Número de músculos testados por membro x 5

Este cálculo foi utilizado no estudo para verificar se houve alteraçóes no percentual de força dos membros avaliados entre a $1^{\mathrm{a}}$ e a $2^{\mathrm{a}}$ avaliação.

\section{Análise Estatística}

A análise estatística foi realizada para caracterizar uma maior confiabilidade da amostra, utilizando o teste de igualdade de duas proporçôes, Wilcoxon, Kruskal-Wallis, Mann-Whitney com intervalo de confiança (95\%) para Média, p-valor e nível de significância de 0,10 (10\%), devido a uma baixa amostragem.

Foram utilizados testes não paramétricos, pois a normalidade da distribuição e a homocedasticidade (homogeneidade das variâncias) não foi observada na amostra.

\section{RESULTADOS}

\section{Caracterizaçáo da populaçáo estudada}

Foram 12 prontuários incluídos, sendo 5 (42\%) do sexo masculino e 7 (58\%) do sexo feminino. A faixa etária da amostra durante o acompanhamento da FM foi de $33 \%$ com idade entre 30 e 40 anos, $42 \%$ com idade entre 40 e 50 anos e $25 \%$ com idade entre 50 e 59 anos de idade.

A maioria dos indivíduos teve poliomielite com idade acima de um ano (67\%). Quanto faixa de idade na qual a SPP foi confirmada, $17 \%$ dos pacientes relataram que foi entre 30 e 40 anos, 50\% entre 40 e 50 anos, e $33 \%$ acima dos 50 anos. Sessenta e sete por cento dos indivíduos relataram início da nova fraqueza em até cinco anos antes da primeira avaliação da FM e 33\% relataram a nova fraqueza a mais de seis anos.

Quanto aos MMSS mais funcionais, foi observado que o membro superior direito (MSD) foi o mais funcional com $67 \%$, comparado ao membro superior esquerdo (MSE) com 33\%. Com relação aos MMII mais funcionais, não houve diferenças percentuais, sendo 50\% para cada membro.

Verificou-se diferença entre a distribuição de sequela aguda e residual para os pacientes Diparéticos, Diplégicos, Quadriplégicos. Nota-se que em pacientes Diplégicos e Quadriplégicos existe maior percentual em sequela aguda ( $42 \%$ e $25 \%$, respectivamente) já em pacientes Diparéticos, o maior percentual é de sequela residual (58\%), ou seja, a maioria dos pacientes estudados apresentou uma diplegia na fase aguda da poliomielite, e na fase residual apresentam uma diparesia.

A fase de reabilitaçáo motora foi outro ponto observado, 17\% dos pacientes realizavam reabilitação antes da $1^{\text {a }}$ avaliação da FM, 33\% só iniciaram reabilitação motora após a $1^{a}$ avaliação da FM, e 50\% dos pacientes não realizava nem iniciou nenhum tipo de reabilitação motora. Com relação à marcha, observamos que a maioria dos pacientes respondeu possuir marcha comunitária, ou seja, $83 \%$ contra $17 \%$ dos pacientes apresentavam marcha domiciliar.

\section{Força Muscular}

No grupo I, foram analisados os músculos extensores de joelho e dorsiflexores de tornozelos, e houve diminuição da FM no membro mais funcional entre a $1^{\text {a }}$ e $2^{\mathrm{a}}$ avaliação do teste $\mathrm{MRC}(\mathrm{p}=0,10)$ e redução da média de 5,0 para 3,7 em ambos. Nos demais grupos musculares, não foram observados variaçóes da FM. Quando os resultados das médias foram observados, após dois anos da $1^{\text {a }}$ avaliaçáo realizada, houve uma pequena reduçáo na média dos músculos abdutores de ombro e igualmente nos músculos extensores de punho de 5,0 para 4,7 ( $\mathrm{p}=0,32)$ e dos músculos flexores de cotovelo não houve diferença da média, se mantendo em 5,0 ( $\mathrm{p}=1,0)$. Ainda nos MMII os músculos flexores do Quadril houve redu- 
ção da média de 4,7 para 4,0 (p=0,16). Sendo a FM testada em cinco dos seis grupos musculares, foi visto que a maior redução da força ocorreu em grupos musculares dos MMII mais funcionais (Gráfico 1). Neste caso também foi aplicada a MRC para verificar a diminuição da FM total em percentual do membro mais funcional, sendo o membro superior de $100 \%$ para $96 \%(\mathrm{p}=0,32)$ e no membro inferior de $98 \%$ para $76 \%(\mathrm{p}=0,10)$.

Quanto ao grupo II, não foram observadas alteraçôes da FM total em nenhum dos seis grupos musculares testados pelo MRC, porém os resultados detalhados mostram que após três anos da $1^{\text {a }}$ avaliação realizada, houve pequena diminuição da FM nos músculos extensores de joelho de 3,0 para 2,7 com $(\mathrm{p}=0,65)$ e um leve aumento da força em abdutores do ombro, flexores de cotovelo de 4,7 para 5,0 com ( $\mathrm{p}=0,32)$ em ambos e flexores de quadril de 2,7 para 3,0 com ( $\mathrm{p}=0,32$; Gráfico 2). Houve aumento no percentual de FM total do membro mais funcional no MS de $96 \%$ para $100 \%(\mathrm{p}=0,32)$, e no MI manteve-se estável.

No grupo III foi analisado a média da FM que após quatro anos da $1^{\text {a }}$ avaliação do MRC realizada, houve uma pequena diminuição da FM em músculos abdutores de ombro de 5,0 para 4,7 ( $\mathrm{p}=0,32)$ e músculos dorsiflexores de tornozelos de 3,0 para 2,7 ( $\mathrm{p}=0,78)$, e leve

\section{Grupo I (Intervalo de 2 anos entre os testes)}

- $12 \mathrm{MRC}$ - $22 \mathrm{MRC}$

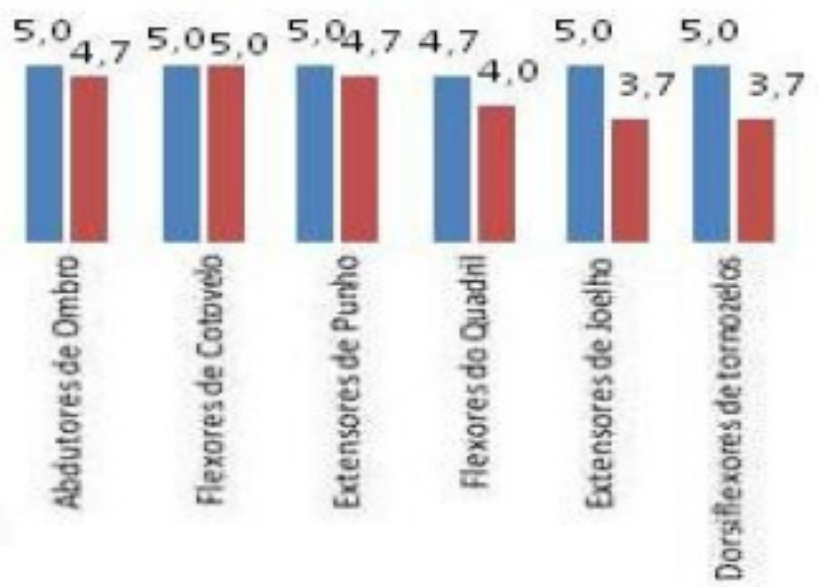

Gráfico 1. Representação gráfica dos resultados das médias da $1^{a}$ e $2^{a}$ $M R C$ de todos os grupos musculares do grupo de indivíduos I. aumento da FM em músculos flexores de quadril de 1,0 para 1,3 (p=0,56; Gráfico 3). Houve diminuição no percentual de FM total do membro mais funcional do MS de $100 \%$ para $98 \%(\mathrm{p}=0,32)$, quando ao MI manteve-se estável em 40\%.

No grupo IV, não foram observadas alteraçóes da FM entre as avaliaçóes do teste MRC. Mas, quando analisados as médias da FM verificou-se que após cinco anos da $1^{\text {a }}$ avaliação do MRC realizada, nos músculos do MS houve pequena diminuição da força no grupo muscular de abdutores de ombro de 5,0 para 4,3 ( $\mathrm{p}=0,16)$, e nos músculos flexores de cotovelo e extensores de punho náo houve alteração mantendo a média de 5,0 durantes os 5 anos. Nos músculos do MI os flexores de quadril e extensores de joelho, em ambos diminui a FM de 4,3 para 4,0 ( $\mathrm{p}=0,32)$, e os músculos dorsiflexores de tornozelos apresentou um aumento na média de FM de 4,0 para 4,7 $(\mathrm{p}=0,32)$, apresentado Gráfico 4. Houve diminuição no percentual de FM total do membro mais funcional do MS de $100 \%$ para $95,3 \%(\mathrm{p}=0,16)$, e no $\mathrm{MI}$ um pequeno aumento no percentual, subindo de $84 \%$ para $84,6 \%$ $(\mathrm{p}=0,32)$.

Para a última análise da FM dos pacientes, consideramos a amostra total do estudo sem segmentar grupo a grupo. Verificou-se que somente no grupo muscular de

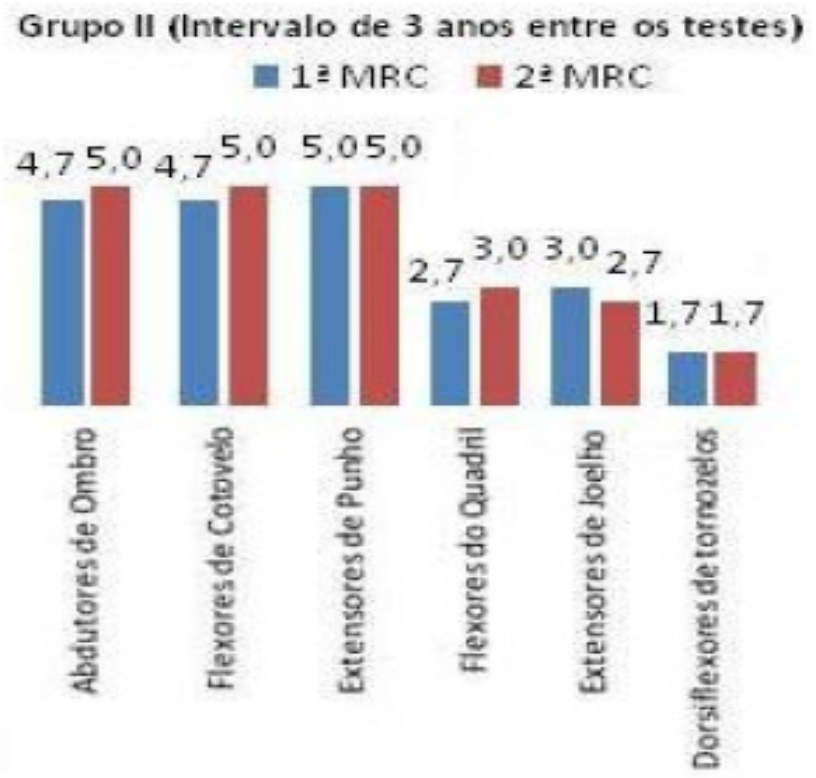

Gráfico 2. Representação gráfica dos resultados das médias da $1^{a}$ e $2^{a}$ $M R C$ de todos os grupos musculares do grupo de individuos II. 


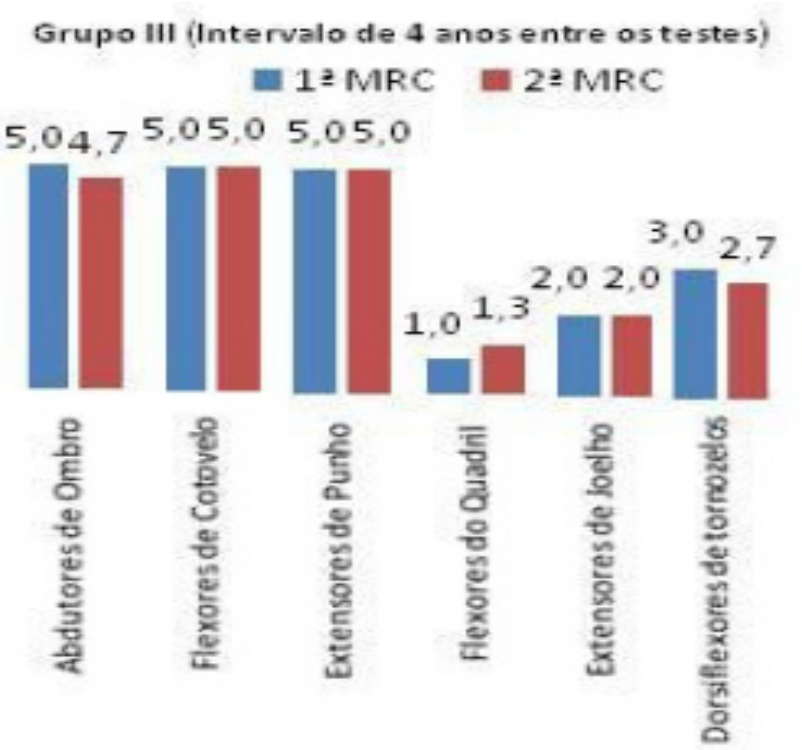

Gráfico 3. Representação gráfica dos resultados das médias da $1^{a}$ e $2^{a}$ $M R C$ de todos os grupos musculares do grupo de individuos III.

extensores de joelho houve redução da média de 3,6 para 3,1 entre a $1^{\mathrm{a}}$ e $2^{\mathrm{a}}$ avaliação do teste MRC ( $\mathrm{p}=0,08$; Tabela 1).

\section{DISCUSSÃO}

Neste estudo foi analisado qual o comportamento da FM nos membros mais funcionais dos pacientes com SPP, visto que estes membros são pouco estudados e nada se encontra na literatura brasileira.

A idade limite de inclusão no presente estudo foi

\section{Grupo IV (Intervalo de 5 anos entre os testes) $=12 \mathrm{MRC}=2 \geq \mathrm{MRC}$}

\section{$5,0 \quad 5,05,05,05,0$}
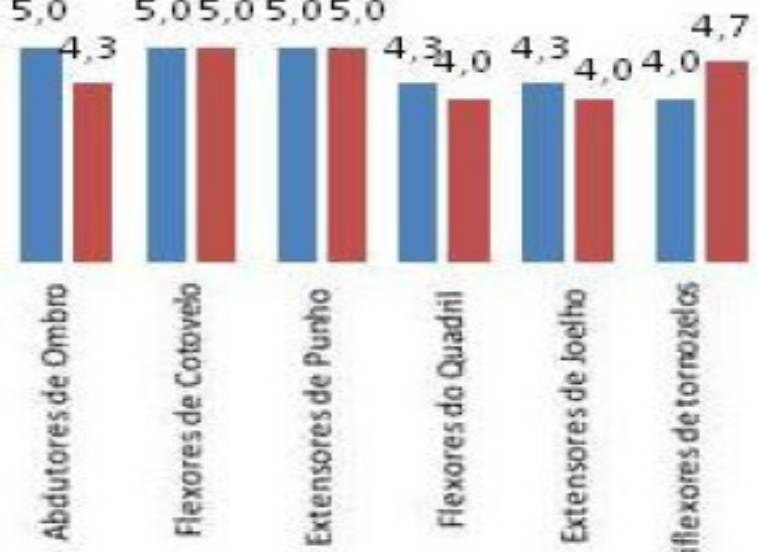

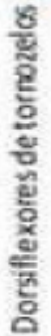

Gráfico 4. Representação gráfica dos resultados das médias da $1^{a}$ e $2^{a}$ $M R C$ de todos os grupos musculares do grupo de individuos $I V$.

de 59 anos com o objetivo de excluir alteraçóes naturais do processo de envelhecimento que ocorrem na terceira idade, como a rápida diminuição da FM. A FM com o envelhecimento na população normal apresentou média de declínio de $1 \%$ ao ano e nos pacientes com SPP o declínio foi de $2 \%{ }^{14}$. Os pacientes com doenças que poderiam causar uma deterioração da FM também foram excluídos.

No presente estudo, foram selecionados pacientes com diversos tipos de sequelas na fase aguda, porém na

Tabela 1. Comparação entre todas as escalas.

\begin{tabular}{|c|c|c|c|c|c|c|c|}
\hline \multicolumn{2}{|c|}{ Escalas } & \multirow{2}{*}{$\begin{array}{c}\text { Média } \\
4,9\end{array}$} & \multirow{2}{*}{$\begin{array}{c}\text { Mediana } \\
5\end{array}$} & \multirow{2}{*}{$\begin{array}{c}\begin{array}{c}\text { Desvio } \\
\text { Padrão }\end{array} \\
0,3 \\
\end{array}$} & \multirow{2}{*}{$\begin{array}{l}\mathrm{N} \\
12 \\
\end{array}$} & \multirow{2}{*}{$\begin{array}{l}\text { IC } \\
0,2 \\
\end{array}$} & \multirow{2}{*}{ p-valor } \\
\hline Abdutores de & 1a. escala & & & & & & \\
\hline & 2a. escala & 4,7 & 5 & 0,5 & 12 & 0,3 & \\
\hline \multirow{2}{*}{$\begin{array}{c}\text { Flexores de } \\
\text { cotovelo }\end{array}$} & 1a. escala & 4,9 & 5 & 0,3 & 12 & 0,2 & \multirow[b]{2}{*}{0,317} \\
\hline & 2a. escala & 5,0 & 5 & 0,0 & 12 & $-x-$ & \\
\hline \multirow{2}{*}{$\begin{array}{l}\text { Extensores de } \\
\text { punho }\end{array}$} & 1a. escala & 5,0 & 5 & 0,0 & 12 & $-x-$ & \multirow[b]{2}{*}{0,317} \\
\hline & 2a. escala & 4,9 & 5 & 0,3 & 12 & 0,2 & \\
\hline \multirow{2}{*}{$\begin{array}{l}\text { Flexores do } \\
\text { Quadril }\end{array}$} & 1a. escala & 3,2 & 3,5 & 1,8 & 12 & 1,0 & \multirow{2}{*}{0,705} \\
\hline & 2a. escala & 3,1 & 3 & 1,8 & 12 & 1,0 & \\
\hline \multirow{2}{*}{$\begin{array}{l}\text { Extensores de } \\
\text { joelho }\end{array}$} & 1a. escala & 3,6 & 4 & 1,6 & 12 & 0,9 & \multirow{2}{*}{0,084} \\
\hline & 2a. escala & 3,1 & 3,5 & 1,8 & 12 & 1,0 & \\
\hline \multirow{2}{*}{$\begin{array}{l}\text { Dorsiflexores de } \\
\text { tornozelo }\end{array}$} & 1a. escala & 3,4 & 4,5 & 2,1 & 12 & 1,2 & \multirow{2}{*}{0,618} \\
\hline & 2a. escala & 3,2 & 4 & 1,9 & 12 & 1,1 & \\
\hline
\end{tabular}

$\mathrm{N}=$ amostra, IC=intervalo de confiança; $\mathrm{x}=$ năo foi possível calcular o IC. 
fase residual ficou evidenciado que todos apresentavam alguma sequela em MMII e nunca exclusivamente em MMSS. Este dado foi muito importante, pois os MMII são duas vezes mais propensos ao aparecimento de novos sintomas de fraqueza muscular em relação aqueles com a nova fraqueza exclusivamente nos MMSS ${ }^{15}$.

Os resultados desse estudo mostram que a sequela residual motora nos indivíduos com SPP é menor comparada à sequela aguda. Este fato sugere que músculos aparentemente não afetados na fase residual podem ter sido acometidos na poliomielite aguda ${ }^{14}$.

Também foi observado o critério de reabilitação, para verificar se os pacientes avaliados realizavam algum tipo de reabilitação motora. E verificou-se que a maioria não realizou reabilitação nem antes e nem após a primeira avaliação da força, o que ajuda a excluir a hipótese de que esses pacientes possam estar realizando algum tipo de esforço inadequado durante a reabilitação.

O foco desse estudo foi os membros mais funcionais pelo fato de serem sobrecarregados para compensar a sequela residual, acabam desenvolvendo uma fraqueza muscular progressiva ${ }^{14,16}$. Em 111 membros clinicamente não afetados de 55 pacientes com SPP, foram estudados, através de teste de FM manual MRC, e comparados com o resultado da eletroneuromiografia, mostrando que grupos musculares desses membros não afetados podem ser potencialmente candidatos à instalação da nova fraqueza muscular ${ }^{16}$.

Foi observado no grupo I, que foi acompanhado por dois anos, que houve tendência para redução da FM em músculos extensores de joelho e dorsiflexores de tornozelos. Pelo fato de haver grandes períodos de até dez anos de estabilidade da FM, não se pode observar grande agravamento da fraqueza muscular por períodos menores que três anos de acompanhamento ${ }^{17}$.

No grupo II, que foi acompanhado por três anos, não foi verificado variaçóes da $\mathrm{FM}$, mas quando se observam os valores das médias, foi encontrado discreto aumento da FM de músculos do MS mais funcional. Talvez se a amostra fosse maior, esses valores poderiam se tornar evidentes, o que nos faria interpretar que após a $1^{\text {a }}$ avaliação seguida de orientações de equipe multidisciplinar, os pacientes economizaram energia e consequentemente depositaram menor carga nos membros. Pacientes acom- panhados no período de três anos, não apresentaram redução da FM, mas aparentemente alguns se apresentam estáveis ou até com certo grau de aumento da $\mathrm{FM}^{17}$.

No grupo III, que foi acompanhado por quatro anos, apesar de não haver diferenças, pode-se observar que houve uma pequena diminuição da FM em apenas dois grupos musculares: que são os abdutores de ombro e dorsiflexores tornozelos, e um leve aumento da força no grupo muscular de flexores de quadril. Este resultado também pode estar relacionado com o período de estabilidade da FM, onde houve diminuição da força. Igualmente ocorreu no grupo IV, que foi acompanhado por cinco anos, ou seja, o grupo de indivíduos que foi acompanhado por um maior período de tempo, também não foi observado variação da FM. Isso nos mostra que pacientes com SPP podem apresentar grandes e variados períodos de estabilidade da FM o que pode interferir no estudo minucioso de acompanhamento da variação da $\mathrm{FM}^{16}$.

Quanto ao objetivo da verificação da FM total em porcentagem no membro mais funcional do paciente no atual estudo, foi para nos dar uma visão mais ampla de quanto às pequenas alteraçóes de variação da FM em grupos musculares isolados interfeririam na FM total do membro. Foi verificado que no MI mais funcional dos grupos II, III e IV, não foram observadas alterações da FM total, permanecendo estável mesmo após cinco anos da $1^{\text {a }}$ avaliação, como ocorrido no grupo IV. Este achado é de grande importância, pois mostra que nesses grupos de indivíduos não houve grande redução da FM dos músculos extensores de joelho como foi encontrado no grupo I.

No grupo I, apesar do intervalo da $1^{\text {a }}$ avaliação para a $2^{\text {a }}$ avaliação ser de apenas dois anos, verificou-se que houve tendência para a redução da força de músculos extensores de joelho e dorsiflexores de tornozelos, este fato pode ter ocorrido pelo fato de que os pacientes que compóe este grupo poderem possuir maior déficit residual, visto que foram incluídos no estudo vários graus de déficit residual, mas a maior prevalência foi de diparesia de MMII. Esta suposição está de acordo com o um estudo encontrado, no qual ficou evidenciado que a capacidade funcional dos indivíduos foi variável e pareceu dependerna maioria das vezes do déficit residual dos pacientes ${ }^{18}$. 
No grupo II, observou-se aumento da FM total como em pacientes acompanhados por três anos, não apresentaram redução da FM, mas aparentemente alguns se apresentaram estáveis ou até com certo grau de aumento da $\mathrm{FM}^{17}$. Já nos grupos I, III, e IV, verificou-se que houve diminuição da FM total do membro em \%. Isto nos mostra que o MS mais funcional perdeu mais força do que o MI mais funcional dos pacientes avaliados. Talvez essa perda possa estar relacionada com a questáo do uso de auxiliares de marcha, como muletas, que possam estar sobrecarregando os MMSS e por isso acarretando em redução da FM total nos membros mais funcionais avaliados.

Como já descrito, todos os pacientes do presente estudo possuem marcha, mesmo que com auxiliares, sendo que a maioria apresenta marcha comunitária, o que evidencia que no mínimo são parcialmente funcionais nos MMII e que provavelmente o gasto de energia desses pacientes durante uma caminhada é alto e poderia estar fortemente relacionado com o grau de nova fraqueza muscular dos $\mathrm{MMII}^{19}$. Porém através dos dados obtidos na avaliação da FM, não foram observados resultados significantes para o aparecimento de nova fraqueza no MI mais funcional quando avaliado por grupo de pacientes. Quando foram comparados todos os grupos de pacientes observou-se redução da FM no músculo extensores de joelho. Este achado pode não ter sido encontrado em cada grupo de indivíduos isoladamente, pelo fato da amostra ser pequena, composta por apenas três pacientes por grupo. Isto porque, alguns estudos encontrados na literatura internacional ${ }^{8,20}$, que possuem maior amostragem acompanharam o comportamento da FM nesta população de pacientes, e chegaram a conclusão que o músculo extensores de joelho foi o que apresentou maior diminuição da FM por ser um dos grupos musculares mais solicitados durante a marcha, sofrendo intensa sobrecarga tanto nos membros mais afetados como nos mais funcionais (menos afetados).

Uma observação importante no atual estudo foi a alta frequência de relatos dos pacientes quanto ao aumento de sensação de cansaço (fadiga). Na prática clínica no SIDNM da UNIFESP/ EPM, os terapeutas têm o desafio de diferenciar o relato de nova fraqueza da sensação de cansaço (fadiga), e devido o paciente apresentar sensação de fadiga excessiva, não sabemos até que ponto essa fadiga relatada pode ser confundida com a sensação de nova fraqueza muscular.

Foi verificada falta de correlação, entre os graus de FM encontradas e a sensação de fraqueza muscular relatada pelos 11 pacientes com SPP do estudo, ou seja, de acordo com o grau de FM encontrado, não foi considerado que houve redução da FM como relatado pelos pacientes durante um período de quatro anos. A fraqueza do músculo quadríceps relatada foi correlacionada com dores no quadril e coxa dos pacientes, mas não com o grau de FM medido ${ }^{21}$. Situação parecida ocorreu no atual estudo, pois todos os pacientes avaliados relataram sentir nova fraqueza, mas não houve resultados significantes para o aparecimento da nova fraqueza. Fato que nos leva a supor que a sensação de nova fraqueza relatada pelos pacientes, possa estar relacionada com algum outro sintoma, como dor muscular ou fadiga.

Também foi visto que em, $20 \%$ dos pacientes com SPP que apresentam dores musculares inexplicadas, a sensação de nova fraqueza é muitas vezes devido a distúrbios mecânicos ${ }^{22}$.

\section{CONCLUSÃO}

Conclui-se que a FM dos pacientes avaliados não apresentou diferenças de variaçóes dentro dos períodos acompanhados. Logo, a queixa relatada pelos pacientes de manifestação da nova fraqueza muscular nos membros mais funcionais pode estar relacionada a outros fatores, como a fadiga, envelhecimento natural ou dores articulares e musculares, e não puramente à diminuição da força de grupos musculares isolados. Quanto à FM total dos membros analisados, conclui-se que as mínimas alterações da FM ocorreram mais no MS mais funcional do que no MI mais funcional. Tendo como base a amostra total do estudo, observou-se diminuição da FM de flexores de joelho, o que sugere a aplicação da presente metodologia em amostras maiores para a confirmação deste achado. Devido às condiçôes de aplicação da MRC no ambulatório de SPP da UNIFESP, no qual vários avaliadores aplicam o teste, o resultado poderá sofrer interfe-rências, pois os avaliadores nem sempre foram os mesmos. Portanto sugere-se que a MRC seja sempre aplicada e reaplicada pelo mesmo avaliador. 


\section{REFERÊNCIAS}

1.Oliveira ASB, Maynard FM. Síndrome Pós-Poliomielite: Aspectos Neurológicos. Rev Neurocienc 2002;10:31-4.

2.Neves MAO, Mello MP, Reis JPB, Rei AM, Antonioli RS, Nascimento OJM, et al. A síndrome pós-polio e o processo de reabilitaçấo motora: relato de caso. Rev Neurocienc 2007;15:321-5.

3.Stibrant Sunnerhagen K, Grimby G. Muscular effects in late polio. Acta Physiol Scand 2001;171:335-40. http://dx.doi.org/10.1046/j.1365$\underline{-201 x .2001 .00836 . x}$

4.Conde MTRP, Oliveira ASB, Quadros AAJ, Moreira GA, Silva HCA, Pereira $\mathrm{RDB}$, et al. Post-polio syndrome: epidemiologic and prognostic aspects in Brazil. Acta Neurol Scand 2009;120:191-7. http://dx.doi.org/10.1111/j.1600-0404.2008.01142.x

5.Dias JMB. Efeitos tardios da poliomielite. Rev Neurocienc 2002;10:35-7.

6.Stolwijk-Swüste JM, Beelen A, Lankhorst GJ, Nollet F, CARPA Study Group. The course of functional status and muscle strength in patients with late-onset sequelae of poliomyelitis: a systematic review. Arch Phys Med Rehabil 2005;86:1693-701. http://dx.doi.org/10.1016/j.apmr.2004.12.022

7.Willén C, Thoren-Jönsson AL, Grimby G, Sunnerhagen KS. Disability in a 4-year follow-up study of people with post-polio syndrome. J Rehabil Med 2007;39:175-80. http://dx.doi.org/10.2340/16501977-0034

8.Grimby G, Stalberg E, Sandberg A, Stibrant Sunnerhagen K. An 8-year longitudinal study of muscle strength, muscle fiber size, and dynamic electromyogram in individuals with late polio. Muscle Nerve 1998;21:1428-37. http://dx.doi.org/10.1002/(SICI) 1097-4598(199811)21:11<1428::AID-MUS10>3.0.CO;2-X

9.Medical Research Council. Aids to the examination of the peripheral nervous system. Edinburgh: WB Saunders, 2000, p 1-2.

10.Berlly MH, Strauser WW, Hall KM. Fatigue in post-polio syndrome. Arch Phys Med Rehabil 1991;72:115-8.

11.Gawne AC, Halstead LS. Post-polio syndrome: pathophysiology and clinical management. Crit Rev Phys Rehabil Med 1995;7:147-88.

12.Fordyce CB, Gagne D, Jalili F, Alab S, Arnold DL, Costa DD, et al. Elevated serum inflammatory markers in post-poliomyelitis syndrome. J Neurol Sci, 2008;271:80-6. http://dx.doi.org/10.1016/j.jns.2008.03.015
13.Scott OM, Hyde SA, Goddard C, Dubowitz V. Quantitation of muscle function in children: A prospective study in Duchenne muscular dystrophy. Muscle Nerve.1982;5:291-301. http://dx.doi.org/10.1002/mus.880050405 14.Oliveira ASB, Quadros AAJ, Silva HCA, Conde MTRP, Silva TM, Fontes SV, et al. Síndrome Pós-Poliomielite (SPP) - Orientaçôes para profissionais de saúde. São Paulo: SES/SP, 2008, 126p.

15.Windebank AJ, Litchy WJ, Daube JR, Kurland LT, Codd MB, Iverson R. Late effects of paralytic poliomyelitis in Olmsted County, Minnesota. Neurology 1991;41:501-7.

16.Chanda MF. Clinical predictors of eletroctromyographic finding of remote pólio in unaffected limbs of adults with a history of acute paralytic poliomyelitis. Presented on Ninth International Post-Polio Health and Ventilator-Assited Living Conference: Stratégies for Living Well. Post-Polio Health 2006;22:1-2. 17.Dalakas MC. The Post polio syndrome as an evolved clinical entily: Definition and Clinical Description. Ann N Y Acad Sci 1995;753:68-80. http:// dx.doi.org/10.1111/j.1749-6632.1995.tb27532.x

18.Dalakas MC, Elder G, Hallett M, Ravits J, Baker M, Papadopoulos N, et al. A long term follow-up study of patients with post-poliomyelitis neuromuscular symptoms. N Engl J Med 1986;314:959-63. http://dx.doi.org/10.1056/ NEJM198604103141505

19.Brehm M A, Nollet F, Harlaar J. Energy demands of walking in persons with postpoliomyelitis syndrome: Relationship with muscle strength and reproducibility. Arch Phys Med Rehabil 2006;87:136-40. http://dx.doi. org/10.1016/j.apmr.2005.08.123

20.Agre JC, Grimby G, Rodriquez AA, Einarsson G, Swiggum ER, Franke TM. A comparison of symptoms between Swedish and American post-polio individuals and assessment of lower limb strength a four-year cohort study. Scand J Rehabil Med 1995;27:183-92.

21.Nordgren B, Falck B, Stalberg E, Ronquist G, Waldenström A, Ahlström $\mathrm{H}$, et al. Postpolio muscular dysfunction: relationships between muscle energy metabolism, subjective symptoms, magnetic resonance imaging, electromyography, and muscle strength. Muscle Nerve 1997;20:1341-51. http://dx.doi.org/10.1002/(SICI) 1097-4598(199711)20:11<1341::AID-MUS1>3.0.CO;2-A

22.Windebank AJ, Litchy W, Daube JR, Iverson RA. Lack of progression of neurological deficit in survivors of paralytic polio: a 5 year prospective population-based study. Neurology 1996;46:80-4. http://dx.doi.org/ 10.1212/ WNL.46.1.80 